\title{
Fadiga por compaixão em enfermeiros de urgência e emergência hospitalar de adultos*
}

\author{
Elisabete Maria das Neves Borges ${ }^{1}$ \\ (D) https://orcid.org/0000-0002-6478-1008 \\ Carla Isabel Nunes da Silva Fonseca ${ }^{2}$ \\ (DiD https://orcid.org/0000-0003-1165-1995 \\ Patrícia Campos Pavan Baptista ${ }^{3}$ \\ (D) https://orcid.org/0000-0003-1433-6456 \\ Cristina Maria Leite Queirós ${ }^{4}$ \\ (1D https://orcid.org/0000-0002-8045-5317 \\ María Baldonedo-Mosteiro 5 \\ (D) https://orcid.org/0000-0001-9729-7119 \\ María Pilar Mosteiro-Diaz ${ }^{6}$ \\ (D) https://orcid.org/0000-0002-3375-9334
}

* Artigo extraído da dissertação de mestrado "Fadiga por compaixão em enfermeiros de urgência hospitalar", apresentada à Escola Superior de Enfermagem do Porto, Porto, Portugal.

${ }^{1}$ Escola Superior de Enfermagem do Porto, Porto, Portugal.

2 Centro Hospitalar de São João, Porto, Portugal.

${ }^{3}$ Universidade de São Paulo, Escola de Enfermagem, Departamento de Orientação Profissional, São Paulo, SP, Brasil.

4 Universidade do Porto, Faculdade de Psicologia e de Ciências da Educação, Porto, Portugal.

5 Universidad de Oviedo, Facultad de Psicología, Oviedo, Espanha.

6 Universidad de Oviedo, Departamento de Medicina, Enfermería, Oviedo, Espanha.
Objetivo: avaliar o nível de fadiga por compaixão em enfermeiros e sua associação em função de características sociodemográficas/profissionais. Método: estudo quantitativo, descritivo e transversal, com 87 enfermeiros de um serviço de urgência e emergência de adultos, de um hospital universitário. Aplicaram-se um questionário sociodemográfico/profissional e a escala Professional Quality of Life Scale 5. Para a análise dos dados, recorreu-se à estatística descritiva e inferencial. Resultados: verificou-se que a satisfação por compaixão apresenta as médias mais elevadas, seguida do burnout e do estresse traumático secundário. Encontraram-se no nível elevado $51 \%$ dos enfermeiros na satisfação por compaixão, $54 \%$ no burnout e $59 \%$ no estresse traumático secundário. Os participantes com mais idade apresentaram médias superiores de satisfação por compaixão, enquanto os do sexo feminino, mais novos, com menos tempo de experiência profissional e que não tinham atividades de lazer evidenciaram média superior de estresse traumático secundário. Conclusão: existe fadiga por compaixão expressa na grande percentagem de enfermeiros com elevados níveis de burnout e de estresse traumático secundário. A fadiga depende de fatores individuais como idade, sexo, experiência profissional e atividades de lazer. A pesquisa e a compreensão desse fenômeno permitem o desenvolvimento de estratégias de promoção de saúde no trabalho.

Descritores: Fadiga por Compaixão; Enfermeiros; Hospitais; Emergências; Esgotamento Profissional; Trabalho.

\section{Como citar este artigo}

Borges EMN, Fonseca CINS, Baptista PCP, Queirós CML, Baldonedo-Mosteiro M, Mosteiro-Diaz MP. Compassion fatigue among nurses working on an adult emergency and urgent care unit. Rev. Latino-Am. Enfermagem. 2019;27:e3175. [Access $\uparrow+1$ ]; Available in: DOI: http://dx.doi.org/10.1590/1518-8345.2973.3175. 


\section{Introdução}

A segurança, a saúde e o bem-estar dos profissionais de saúde são alvo de atenção a nível mundial, dadas as exigências emocionais da sua tarefa, com significativa importância para a produtividade, a competitividade e a sustentabilidade das organizações ${ }^{(1-3)}$.

Considerada uma das maiores ameaças à saúde mental dos profissionais de saúde ${ }^{(4-7)}$, a fadiga por compaixão foi definida como "comportamentos e emoções consequentes naturais resultantes do conhecimento de um evento traumatizante experimentado por um outro significativo - o stress resultante do ajudar ou do querer ajudar uma pessoa traumatizada ou em sofrimento"(8). Posteriormente, o modelo de Qualidade de Vida Profissional apresenta a fadiga por compaixão como resultado da combinação de elevado burnout, estresse traumático secundário e reduzida satisfação por compaixão(9).

Diferentes fatores contribuem para a fadiga por compaixão, destacando-se a nível individual a personalidade, a educação, a experiência de trabalho, a qualidade de vida pessoal, e a nível organizacional a especificidade da função e a reforma do sistema de saúde ${ }^{(6,10)}$. Pela sua exigência e pelo contato frequente com situações traumáticas, o trabalho de enfermagem nos contextos de urgência e emergência torna os enfermeiros suscetíveis a interiorizarem a dor dos seus pacientes, potenciando a fadiga por compaixão(11-13).

As manifestações da fadiga por compaixão são variadas e nem sempre têm sido valorizadas. Desenvolvemse ao longo do tempo, comprometendo não só a saúde física, psicológica, cognitiva e espiritual dos profissionais, mas também a sua vida pessoal, social e profissional, o que se repercute negativamente no seu bem-estar e na qualidade de vida, bem como nas próprias instituições de saúde e na qualidade dos cuidados prestados ${ }^{(4-5,14-16)}$. Tendo os enfermeiros uma atividade emocionalmente exigente realizada em condições estressantes ${ }^{(17-20)}$, com este estudo pretendeu-se avaliar o nível de fadiga por compaixão em enfermeiros de um serviço de urgência e emergência hospitalar de adultos e sua associação em função de características sociodemográficas e profissionais.

\section{Método}

Trata-se de um estudo quantitativo, descritivo e transversal, desenvolvido de maio a julho de 2017, com enfermeiros portugueses de um serviço de urgência e emergência de adultos de um hospital universitário da cidade do Porto, Portugal. Definiu-se como critério de inclusão ser enfermeiro com tempo de serviço superior a 6 meses. Optou-se por uma amostra de conveniência a partir de uma população de 93 enfermeiros. Participaram da pesquisa 87 enfermeiros, o que representou uma taxa de adesão de $94 \%$.

A coleta de dados foi efetuada por meio de um instrumento de auto-preenchimento, de caraterização sociodemográfica (sexo, idade, estado civil, ter filhos, habilitações acadêmicas e atividade de lazer) e profissional (tempo de experiência profissional, vínculo de trabalho, horário de trabalho, ter dependentes a cargo, renda mensal e considerar o seu trabalho estressante), e da Professional Quality of Life Scale - ProQOL5, traduzida e adaptada para a população portuguesa ${ }^{(9,21)}$. Este instrumento avalia a fadiga por compaixão, sendo constituído por 30 itens, divididos em 3 subescalas, cada uma formada por 10 itens e que avalia os três fenômenos distintos da satisfação por compaixão, burnout e estresse traumático secundário. Cada item tem uma afirmação à qual se atribui uma pontuação numa escala de likert, que varia de 1 (Nunca) a 5 (Muito frequentemente). A fadiga por compaixão resulta de elevado burnout e elevado estresse traumático secundário. Optou-se por essa escala, pois tem sido atualmente uma das mais utilizadas para avaliar a fadiga por compaixão, sendo do interesse dos investigadores, uma vez que integra o componente positivo de satisfação por compaixão e não apenas o componente negativo(9).

Esta pesquisa foi aprovada pela Comissão de Ética para a Saúde, pelo Conselho de Administração do hospital universitário e pelos autores da versão portuguesa da Professional Quality of Life Scale - ProQOL5. O estudo encontra-se integrado no projeto "INT-SO Dos contextos de trabalho à saúde ocupacional dos profissionais de enfermagem, um estudo comparativo entre Portugal, Brasil e Espanha", do NursID: Inovação e Desenvolvimento em Enfermagem: Center for Health Technology and Services Research (CINTESIS).

Após contato informal com o enfermeiro chefe do serviço de urgência e emergência de adultos, onde foi realizada a pesquisa, foi agendado o momento de aplicação dos instrumentos. Através do contato direto por uma das investigadoras com os enfermeiros potenciais participantes, foi apresentado o documento de informação do estudo, o consentimento informado e o instrumento de coleta de dados. Com os enfermeiros que aceitaram participar da pesquisa, foi combinada a devolução dos instrumentos, passadas duas semanas, em envelope fechado, de forma a garantir o anonimato, sendo recolhidos pela mesma investigadora.

Os dados foram analisados por meio da estatística descritiva e inferencial através do Statistical Package for the Social Sciences, versão 24. Realizou-se a análise com recurso a frequências absolutas e relativas e a medidas de tendência central como a média, mediana, máximo, mínimo e o Desvio Padrão, o coeficiente de correlação de Pearson, o teste paramétrico t de student 
para amostras independentes e o teste não paramétrico de Mann-Whitney. Na análise estatística, assumiu-se o limite de significância de $\mathrm{p}<0,05$ (grau de confiança de $95 \%$ ). Avaliaram-se a confiabilidade das subescalas através do coeficiente alfa de cronbach e a normalidade através do teste de aderência de Kolmogorov-Smirnov.

Para o cálculo dos pontos de corte da Professional Quality of Life Scale - ProQOL5, foram seguidas as orientações da autora(9), transformando os valores primários das subescalas satisfação por compaixão, burnout e estresse traumático secundário em Zscores e estes em tscores. A reconversão dos valores primários forçada para obter $M=50$ e $D P=10$ possibilita a comparação entre os valores das três dimensões e a comparação com outros estudos.

\section{Resultados}

Em relação às características sociodemográficas e profissionais, $57(65,5 \%)$ enfermeiros eram do sexo feminino, com média de idade de 37,1 ( $D P=6,3$ ) anos, sendo a mediana de 36, a mínima de 25 e a máxima de 52 anos, 50 (57,5\%) não tinham parceiro, 42 (48,2\%) tinham filhos, $80(94,1 \%)$ eram licenciados e $5(5,6 \%)$ tinham pós-graduação, $84(96,6 \%)$ tinham vínculo definitivo, 85 $(97,7 \%)$ trabalhavam por turnos rotativos, tinham 13,9
$(\mathrm{DP}=6,1)$ anos de média de experiência profissional, com um mínimo de 3, máximo de 31 e mediana de 11 anos, $55,2 \%$ sem dependentes a cargo e 12,6\% dependiam apenas da sua renda mensal. Quanto ao estresse no local de trabalho, $86(96,6 \%)$ participantes consideraram o seu trabalho estressante, apesar de $56(64,4 \%)$ terem algum tipo de atividade de lazer fora do horário de trabalho, sendo a atividade mais citada a prática de exercício físico.

A Professional Quality of Life Scale - ProQOL5 apresentou valores de coeficiente de alfa de Cronbach referentes à satisfação por compaixão $(0.90)$, burnout (0.77) e ao estresse traumático secundário (0.82) semelhantes aos obtidos pela versão original(9) e portuguesa(21), com valores de $0,88,0,75,0,81$ e 0,86 , $0,71,0,83$, respetivamente.

A análise das dimensões da Qualidade de vida profissional revela que a satisfação por compaixão apresenta os valores médios mais elevados, seguida do burnout, tendo o estresse traumático secundário os valores mais baixos (Tabela 1). A análise de correlação entre as dimensões revelou que a correlação entre a satisfação por compaixão e o burnout é negativa e forte, enquanto entre a satisfação por compaixão e o estresse traumático secundário é negativa, mas fraca, e entre burnout e estresse traumático secundário é positiva, mas fraca (Tabela 1).

Tabela 1 - Análise descritiva e correlacional das dimensões da fadiga por compaixão dos enfermeiros, Porto, Portugal, 2017

\begin{tabular}{|c|c|c|c|c|c|c|}
\hline Dimensões da ProQOL5* $(10-50)$ & Min & Mx & $M$ & DP & Satisfação por compaixão & Burnout \\
\hline Satisfação por compaixão & 22 & 48 & 37,1 & 5,9 & & \\
\hline Burnout & 16 & 38 & 26,0 & 5,6 & $-0,602(0.000)^{*}$ & \\
\hline Estresse traumático secundário & 11 & 39 & 23,9 & 5,5 & $-0,116$ & $0,456(0,000)^{\dagger}$ \\
\hline
\end{tabular}

*ProQOL5 = Professional Quality of Life Scale; ${ }^{\dagger}$ valor obtido por meio do coeficiente de correlação de Pearson

Com base nos pontos de corte, verificou-se (Tabela 2) que $51 \%$ dos enfermeiros têm nível alto de satisfação por compaixão e $20 \%$ um nível baixo, no burnout 54\% têm nível alto e $24 \%$ nível baixo e no estresse traumático secundário
$59 \%$ têm nível alto e $20 \%$ nível baixo. Agrupando os níveis médio/alto das diferentes subescalas, encontramos na satisfação por compaixão $81 \%$, no burnout $76 \%$ e no estresse traumático secundário $80 \%$ dos participantes.

Tabela 2 - Pontos de Corte da Professional Quality of Life Scale - ProQOL5 e frequências dos níveis das subescalas satisfação por compaixão, burnout e estresse traumático secundário dos enfermeiros, Porto, Portugal, 2017

\begin{tabular}{|c|c|c|c|c|c|c|}
\hline \multirow{2}{*}{$\begin{array}{l}\text { Dimensões Professional Quality of Life } \\
\text { Scale - ProQOL5* }\end{array}$} & \multicolumn{3}{|c|}{ Pontos de Corte - Percentis (tscores) } & \multicolumn{3}{|c|}{ Níveis - N (\%) } \\
\hline & 25 & 50 & 75 & Baixo & Médio & Alto \\
\hline Satisfação por compaixão & 42,9 & 51,4 & 56,4 & $17(19,5)$ & $26(29,9)$ & $44(50,6)$ \\
\hline Burnout & 42,8 & 48,1 & 58,8 & $21(24,1)$ & $19(21,8)$ & $47(54,0)$ \\
\hline Estresse traumático secundário & 43,0 & 48,3 & 59,1 & $17(19,5)$ & $19(21,8)$ & $51(58,6)$ \\
\hline
\end{tabular}

*ProQOL5 = Professional Quality of Life Scale

Considerando as características sociodemográficas e profissionais, verificou-se que dos $51 \%$ que apresentam nível alto de satisfação por compaixão, a maioria são mulheres $(53 \%)$, com idade superior ou igual a 36 anos (59\%), sem parceiro (56\%), licenciada (50\%), com experiência profissional inferior a 11 anos (53\%), tempo de experiência no serviço inferior a 9 anos (54\%) e considera o seu trabalho estressante (51\%).

Apresentaram nível alto de burnout $54 \%$ dos enfermeiros, sendo sobretudo mulheres (54\%), com idade inferior a 35 anos (61\%), sem parceiro (58\%), pós Graduados/Mestrados/Doutorados (60\%), com experiência profissional superior ou igual a 12 anos (55\%), tempo de experiência no serviço superior ou igual a 10 anos $(64 \%)$ e que consideram o trabalho estressante $(55 \%)$.

Dos $59 \%$ com nível alto de estresse traumático secundário, a maioria são mulheres $(67 \%)$, com idade inferior a 35 anos (74\%), sem parceiro (64\%), licenciada (61\%), com experiência profissional inferior a 11 anos 
(68\%), tempo de experiência no serviço inferior a 9 anos (69\%) e considera o trabalho estressante (60\%).

A análise comparativa em função de características sociodemográficas e profissionais revelou diferenças estatisticamente significativas apenas em função do grupo etário, sexo, experiência profissional e atividades de lazer (Tabela 3 ). Em relação ao grupo etário, os enfermeiros de idade igual ou superior a 36 anos apresentaram médias superiores de satisfação por compaixão e inferiores de burnout. Os enfermeiros mais novos, do sexo feminino e com experiência igual ou inferior a 11 anos evidenciaram média superior de estresse traumático secundário. Por fim, os que não tinham atividades de lazer apresentaram médias superiores de burnout e de estresse traumático secundário.

Tabela 3 - Análise comparativa da ProQOL5* em função da idade, sexo e atividades de lazer dos enfermeiros, Porto, Portugal, 2017

\begin{tabular}{|c|c|c|c|c|}
\hline ProQOL5* & Variável & $\mathbf{N}$ & $M(D P)$ & $\mathbf{p}^{\dagger}$ \\
\hline \multirow{2}{*}{ Satisfação por compaixão } & $\leq 35$ anos & 38 & $46,7(10,7)$ & \multirow{2}{*}{$-0,006$} \\
\hline & $\geq 36$ anos & 49 & $52,5(8,6)$ & \\
\hline \multirow{2}{*}{ Burnout } & Atividade Lazer -Sim & 56 & $48,3(9,2)$ & \multirow{2}{*}{0,041} \\
\hline & Atividade Lazer -Não & 31 & $52,9(10,7)$ & \\
\hline \multirow{8}{*}{$\begin{array}{l}\text { Estresse traumático } \\
\text { Secundário }\end{array}$} & $\leq 35$ anos & 38 & $53,1(9,8)$ & \multirow{2}{*}{0,008} \\
\hline & $\geq 36$ anos & 49 & $47,5(9,4)$ & \\
\hline & Mulher & 57 & $51,9(9,1)$ & \multirow{2}{*}{0,011} \\
\hline & Homem & 30 & $46,2(10,6)$ & \\
\hline & $\leq 11$ anos & 38 & $52,6(10,6)$ & \multirow{2}{*}{0,031} \\
\hline & $\geq 12$ anos & 49 & $47,9(9,0)$ & \\
\hline & Atividade Lazer -Sim & 56 & $48,3(9,4)$ & \multirow{2}{*}{0,041} \\
\hline & Atividade Lazer -Não & 31 & $52,9(10,4)$ & \\
\hline
\end{tabular}

*ProQOL5 = Professional Quality of Life Scale; ${ }^{\text {} v a l o r ~ o b t i d o ~ p o r ~ m e i o ~ d o ~ t e s t e ~} \mathrm{t}$ de Student

Não foram encontradas diferenças em função do estado civil, da existência de filhos, do grau acadêmico, do tempo de experiência no serviço, dos dependentes a carga, da renda familiar e da percepção do trabalho como estressante.

\section{Discussão}

Os valores médios encontrados através dos escores brutos para as subescalas satisfação por compaixão, burnout e estresse traumático secundário são semelhantes aos de outras investigações ${ }^{(22-23)}$, o mesmo acontecendo com os valores da satisfação por compaixão, burnout e estresse traumático secundário em função dos pontos de corte $e^{(4,9,21)}$. De um modo geral, os resultados deste estudo demonstram, como nos estudos supracitados, a predisposição que os enfermeiros têm para desenvolver níveis elevados de fadiga por compaixão. Diversas pesquisas demonstraram os custos emocionais de cuidar de pessoas em sofrimento, realçando a associação entre a fadiga por compaixão e o estresse no trabalho, sobretudo quando este é crônico e se transforma em burnout ${ }^{(18,24-26)}$, bem como quando as situações são emocionalmente desgastantes, podendo conduzir ao estresse-pós-traumático primário(10,14-15,20,27). De fato, a possibilidade de poderem ser afetados pelas suas vivências, associada ao carácter altruísta e à preocupação empática que caracteriza a relação profissional estabelecida com os pacientes, constituem fatores de risco para o desenvolvimento da fadiga por compaixão e, consequentemente, uma ameaça à saúde mental e ao bem-estar dos enfermeiros ${ }^{(6-7)}$.

Relativamente à variação dos níveis de fadiga por compaixão em função de características sociodemográficas e profissionais, os dados obtidos corroboram pesquisas em que as mulheres apresentam médias superiores aos homens no estresse traumático secundário, talvez pela capacidade empática daquelas de se identificarem com os seus pacientes e absorverem os seus medos e traumas(9). Contudo, o mesmo não acontece em função da idade, que em outros estudos não revelou uma diferença significativa ${ }^{(9,21-22)}$, já que nesta pesquisa os enfermeiros com idade igual ou superior a 36 anos apresentaram médias superiores de satisfação por compaixão, mas inferiores de estresse traumático secundário. Além disso, os profissionais mais novos revelaram médias inferiores de satisfação por compaixão e superiores de estresse traumático secundário, resultados semelhantes aos de outros investigadores(28), maior capacidade de adaptação às situações, bem como pelo mito do trabalhador saudável, ou seja, os enfermeiros que estão efetivamente com malestar psicológico não se voluntariam para a participação em estudos ou já poderão ter abandonado a profissão.

Verificou-se ainda que os enfermeiros com mais idade, em especial as mulheres, apresentaram maiores níveis de satisfação por compaixão, o que corrobora resultados de outros estudos(22), sugerindo que as mulheres evidenciaram maior prevalência na satisfação por compaixão e na capacidade de desempenhar o trabalho ao cuidar de quem sofre. Os enfermeiros com tempo de 
experiência igual ou inferior a 11 anos revelaram valores superiores de estresse traumático secundário, o que se deve provavelmente ao fato de serem menos experientes e se identificarem com mais facilidade com os pacientes. Tal fator sugere, assim, que a fadiga por compaixão diminui com os anos de experiência profissional(7,29), podendo estar relacionada com a capacidade de adaptação que ainda não é tão notória nos enfermeiros menos experientes. Constatou-se que os enfermeiros que não realizavam atividades de lazer estavam mais expostos ao burnout e ao estresse traumático secundário, o que vem confirmar a ideia de que os profissionais que não investem na sua qualidade de vida pessoal estão em maior risco de fadiga por compaixão(6), pois concentram todo o seu foco de vida no trabalho. Assim, quando não correspondem às expectativas, esses enfermeiros ficam mais vulneráveis ao burnout e ao mal-estar psicológico.

Apesar do estudo apresentar como limitações o seu carácter transversal e a amostra de conveniência, o que não permite a extrapolação dos resultados para outros contextos, pode contribuir para o estudo da fadiga por compaixão como fenômeno sobre o qual tem crescido a evidência científica de que é uma ameaça à saúde mental dos enfermeiros ${ }^{(5,7)}$. Dessa forma, é possível alertar os enfermeiros e gestores hospitalares de que é importante monitorizar a saúde mental dos profissionais de saúde, para que o seu estado emocional e psicológico não seja demasiado afetado pelos cuidados que prestam aos doentes, podendo manter um nível ótimo de desempenho ao nível dos cuidados prestados.

\section{Conclusão}

O estudo permitiu verificar que existe satisfação por compaixão, burnout e estresse traumático secundário, com níveis médios a altos nesta amostra, e que a fadiga por compaixão depende de fatores pessoais como idade, sexo, experiência profissional e atividades de lazer.

Consideramos que a pesquisa e a compreensão desse fenômeno potencializam o desenvolvimento de estratégias de promoção de saúde no local de trabalho, em busca de uma melhor qualidade de vida profissional dos enfermeiros e de uma prestação de cuidados de qualidade. Recentemente, diferentes autores enfatizaram as consequências negativas do cuidar dos outros sem cuidar de si, alertando para a necessidade de melhor articular trabalhador e tarefa na promoção da sua saúde ocupacional, tal como vem sendo preconizado na enfermagem do trabalho. Além disso, poderão ser utilizadas, como estratégias de prevenção do estresse ocupacional nos enfermeiros, a implementação de formações sobre sintomas de estresse laboral e fadiga, o estímulo à monitorização regular (ex: através de breves questionários da responsabilidade dos serviços de saúde ocupacional) e os mecanismos de partilha e de suporte entre pares (ex: sessões de discussão de casos reais com partilha de vivências, devendo existir o máximo cuidado em não constituírem momentos de fragilidade pessoal passíveis de acusações profissionais), os quais podem constituir importantes contributos para a prevenção do burnout e da fadiga de compaixão, aumentando a satisfação com a prestação de cuidados aos utentes.

\section{Referências}

1. Elliott C. Emotional labour: learning from the past, understanding the present. British Journal of Nursing. Mark Allen Group; 2017 Oct 26; 26(19):1070-7. doi:10.12968/bjon.2017.26.19.1070.

2. Ueno LGS, Bobroff MCC, Martins JT, Bueno RCRM, Linares PG, SG. Occupational Stress: Stressors Referred By the Nursing Team. J Nurs UFPE on line. 2017; 11(4):1632-8. doi:10.5205/reuol.9763-85423-1-SM.1104201710.

3. Giménez-Espert MC, Prado-Gascó VJ, Valero-Moreno S. Impact of work aspects on communication, emotional intelligence and empathy in nursing. Rev. Latino-Am. Enfermagem. 2019; 27: e3118. doi: http://dx.doi. org/10.1590/1518-8345.2933.3118.

4. Adimando A. Preventing and Alleviating Compassion Fatigue Through Self-Care: An Educational Workshop for Nurses. J Holist Nurs. 2017. doi: $10.1177 / 0898010117721581$.

5. Wentzel D, Brysiewicz P. Integrative Review of Facility Interventions to Manage Compassion Fatigue in Oncology Nurses. Oncol Nurs Forum. 2017; 44(3): E124-40. doi: 10.1188/17.ONF.E124-E140.

6. Pehlivan T. Compassion Fatigue: The Known, Unknown. J Psychiatric Nurs. 2018; 9(2): 129-34. Doi:10.14744/ phd.2017.25582

7. Missouridou E. Secondary posttraumatic stress and nurses' emotional responses to Patient's trauma. J Trauma Nurs. 2017; 24(2):110-5. doi:10.1097/ jtn.0000000000000280.

8. Figley CR. Compassion fatigue: psychotherapists' chronic lack of self care. J Clin Psychol. 2002; 58:1433-41. doi: $10.1002 / j c l p .10090$.

9. Stamm BH. The Concise ProQOL Manual. 2nd ed. Pocatello, ID: ProQOL.org. 2010. Available from: https://www.proqol. org/uploads/ProQOL_Concise_2ndEd_12-2010.pdf

10. Duarte J, Pinto-Gouveia J. The role of psychological factors in oncology nurses' burnout and compassion fatigue symptoms. Eur J Oncol Nurs. 2017; 28:114-21. doi: https://doi.org/10.1016/j.ejon.2017.04.002

11. Van Mol MMC, Kompanje EJO, Benoit DD, Bakker J, Nijkamp MD. The prevalence of compassion fatigue and burnout among healthcare professionals in intensive 
care units: A systematic review. PLoS One. 2015; 10(8):1-22. doi:10.1371/journal.pone.0136955.

12. Drury V, Craigie M, Francis K, Aoun S, Hegney DG. Compassion satisfaction, compassion fatigue, anxiety, depression and stress in registered nurses in Australia: Phase 2 results. J Nurs Manage. 2014; 22(4):519-31. doi:10.1111/jonm.12168.

13. Henson JS. When Compassion Is Lost. Medsurg Nurs. 2017; 26(2):139-142. Available from: https:// www.ncbi.nlm.nih.gov/pubmed/30304598

14. Sinclair S, Raffin-Bouchal S, Venturato L, MijovicKondejewski J, Smith-MacDonald L. Compassion fatigue: A meta-narrative review of the healthcare literature. Int J Nurs Studies. 2017 Apr; 69:9-24. doi.org/10.1016/j. ijnurstu.2017.01.003.

15. Jarrad R, Hammad S, Shawashi T, Mahmoud N. Compassion fatigue and substance use among nurses. Annals Gen Psychiatry. 2018 Mar 13;17(1). Available from: http://dx.doi.org/10.1186/s12991-018-0183-5

16. Ames M, Salmond E, Holly C, Kamienski M. Strategies that reduce compassion fatigue and increase compassion satisfaction in nurses. JBI Database Syst Rev Implement Reports. 2017;15(7):1800-4. doi: 10.11124/JBISRIR-2016-003142.

17. Guirardello EB. Impacto do ambiente de cuidados críticos no burnout, percepção da qualidade do cuidado e atitude de segurança da equipe de enfermagem. Rev. Latino-Am. Enfermagem. 2017; 25: e2884. doi: http:// dx.doi.org/10.1590/1518-8345.1472.2884.

18. Gómez-Urquiza JL, De la Fuente-Solana EI, Albendín-García L, Vargas-Pecino C, Ortega-Campos EM, Cañadas-De la Fuente GA. Prevalence of Burnout Syndrome in Emergency Nurses: A Meta-Analysis. Critical Care Nurse. 2017 Oct; 37(5):e1-e9. doi: http:// dx.doi.org/10.4037/ccn2017508.

19. Puerto J, Soler, L, Montesinos, M, Marcos, A, Chorda, V. A new contribution to the classification of stressors affecting nursing professionals. Rev. Latino-Am. Enfermagem. 2017; 25: e2895-2017. doi:10.1590/15188345.1240.2895.

20. Hinderer KA, VonRueden KT, Friedmann E, McQuillan $K A$, Gilmore R, Kramer B, et al. Burnout, compassion fatigue, compassion satisfaction, and secondary traumatic stress in trauma nurses. J Trauma Nurs. 2014; 21(4):160-9. doi:10.1097/jtn.0000000000000055.

21. Carvalho P, Sá L. Qualidade de vida profissional nos cuidados paliativos: Adaptação Cultural e estudo de validade da escala "Professional Quality of Life 5 (ProQOL5)." Inst Ciências da Saúde da Univ Católica Port. 2011. Available from: http://hdl.handle. net/10400.14/8918

22. Duarte J. Professional quality of life in nurses: Contribution for the validation of the Portuguese version of the Professional Quality of Life Scale-5 (ProQOL-5). Anal Psicol. 2017 Dec 26;35(4):529-42. Available from: http://dx.doi.org/10.14417/ap.1260.

23. Hunsaker S, Chen HC, Maughan D, Heaston S. Factors That Influence the Development of Compassion Fatigue, Burnout, and Compassion Satisfaction in Emergency Department Nurses. J Nurs Scholarship. 2015; 47(2):186-94. doi: 10.1111/jnu.12122.

24. Hooper C, Craig J, Janvrin DR, Wetsel MA, Reimels E. Compassion Satisfaction, Burnout, and Compassion Fatigue Among Emergency Nurses Compared With Nurses in Other Selected Inpatient Specialties. J Emergency Nurs. 2010; 36(5):420-7. doi: 10.1016/j. jen.2009.11.027.

25. Giorgi F, Mattei A, Notarnicola I, Petrucci C, Lancia L. Can sleep quality and burnout affect the job performance of shift-work nurses? A hospital cross-sectional study. J Adv Nurs. 2018 Nov 22; 74(3):698-708. doi:10.1111/ jan. 13484 .

26. Chen S-C, Chen C-F. Antecedents and consequences of nurses' burnout. Manage Decision. 2018 Apr 9;56(4):777-92. doi:10.1108/MD-10-2016-0694.

27. Yu H, Jiang A, Shen J. Prevalence and predictors of compassion fatigue, burnout and compassion satisfaction among oncology nurses: A cross-sectional survey. Int J Nurs Studies. 2016; 57:28-38. doi:10.1016/j. ijnurstu.2016.01.012.

28. Sacco TL, Ciurzynski SM, Harvey ME, Ingersoll GL. Compassion Satisfaction and Compassion Fatigue Among Critical Care Nurses. Crit Care Nurse. 2015; 35(4):32-43. doi:10.4037/ccn2015392.

29. Mooney C, Fetter K, Gross BW, Rinehart C, Lynch C, Rogers FB. A Preliminary Analysis of Compassion Satisfaction and Compassion Fatigue with Considerations for Nursing Unit Specialization and Demographic Factors. J Trauma Nurs. 2017; 24(3):158-63. doi:10.1097/ jtn.0000000000000284.
Autor correspondente:

Elisabete Maria das Neves Borges

E-mail: elisabete@esenf.pt

(D) https://orcid.org/0000-0002-6478-1008
Recebido: 15.01.2019

Aceito: 10.03 .2019
Copyright @ 2019 Revista Latino-Americana de Enfermagem Este é um artigo de acesso aberto distribuído sob os termos da Licença Creative Commons CC BY.

Esta licença permite que outros distribuam, remixem, adaptem e criem a partir do seu trabalho, mesmo para fins comerciais, desde que Ihe atribuam o devido crédito pela criação original. É a licença mais flexível de todas as licenças disponíveis. É recomendada para maximizar a disseminação e uso dos materiais licenciados. 